\title{
Development of Safety Protocol, Features, and Fail-Safes for a Laboratory- Scale Manufacturing Process
}

\section{Ms. Nikki Larson, Western Washington University}

After receiving my bachelor degree in Mechanical Engineering from Bradley University, I started working for Boeing. While at Boeing I worked to receive my master's degree in Mechanical Engineering with an emphasis in Materials and Manufacturing. After leaving Boeing I spent several years in equipment research and development at Starbucks Coffee Company.

From there I decided my heart lied in teaching and left Starbucks to teach Materials Science Technology at Edmonds Community College. I eventually moved to Western Washington University where I have been faculty in the Plastics and Composites Engineering Program (formerly Plastics Engineering Technology) for the past 10 years. My research interests are in composite manufacturing.

\section{Cecile Grubb, Western Washington University}




\title{
Development of Adequate Safety Protocol, Features and Fail-Safes for a Laboratory Scale Manufacturing Process
}

\begin{abstract}
The manufacturing of composite materials has many associated hazards - health, physical, fire/explosive, and environmental hazards, to name a few. With general laboratory safety training and common sense, some hazards are readily apparent and easily detected by the students working on a project. However, many of the dangers associated with volatile or toxic chemicals cannot be seen and consequently go unnoticed until an incident occurs.

This document details the work done by undergraduate students, faculty and staff at Western Washington University to develop appropriate safety features for a laboratory scale solventbased prepreg manufacturing machine. This manufacturing process has a variety of associated hazards including physical hazards (moving parts on the machine), health hazards (use of toxic/sensitizing chemicals), fire/explosive hazards (volatilization of organic solvents during manufacturing process), and environmental hazards (use of ecologically harmful materials), making it an excellent case study for the development of safety protocol by an academic institution. To date there has not been any documentation centered around the challenges associated with creating a solvent-based prepreg treater system. The safety feature and protocol development process is explained in detail and may serve useful for other institutions undertaking similar projects.
\end{abstract}

\section{Introduction}

As part of on-going research into new materials for aerospace composites, Western Washington University faculty, staff and students designed, developed and qualified a laboratory scale solvent-based prepreg manufacturing machine. This machine, commonly referred to as a prepreg treater, is used to replicate the industrial solvent-based prepreg manufacturing process on the small scale. Prepreg is the term used for a fiber reinforcement that has been pre-impregnated with a resin matrix. ${ }^{1}$ The manufacture of prepreg is of interest for both research and teaching purposes, although thus far usage of the prepreg treater has not been incorporated into curriculum.

Throughout the development of the treater and its ongoing usage, safety has been of the upmost importance to the involved students, faculty and staff. The engineering curriculum at Western Washington University places an emphasis on hands-on technical experiences, yielding research students well prepared to recognize the physical hazards associated with industrial machinery. Students, as well as faculty and staff, have found identifying the hazards associated with chemical processes challenging, as these dangers can remain virtually invisible until an accident occurs. As the prepreg treater combines the physical hazards of a complex machine with the health, fire and environmental hazards of a chemical process, increased vigilance when developing safety protocols, features and fail-safes was required for this project. To date there 
has not been any documentation centered around the challenges associated with creating a solvent-based prepreg treater system.

As an employer in Washington State, workplace health and safety at Western Washington University are governed by WISHA, the Washington Industrial Safety and Health Act of 1973, and are enforced by the state agency DOSH, the Division of Occupational Safety and Health. ${ }^{2,3}$ The State of Washington is allowed by the federal government to use these regulations in place of OSHA (the federal Occupational Safety and Health Administration), which gives the state the ability to have more stringent rules than federally required where needed. ${ }^{4}$ Throughout the course of this project, the research team worked with Western Washington University's Environmental

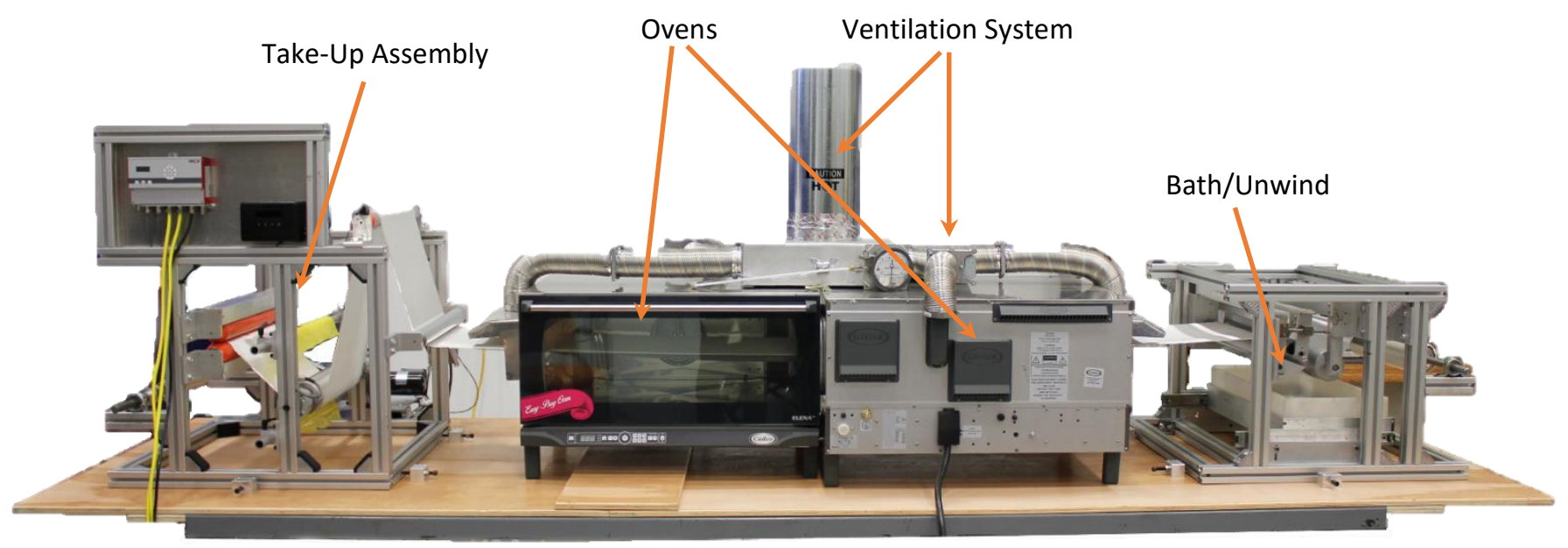

Figure 1. Laboratory scale solvent-based prepreg manufacturing machine built by Western Washington University

Health and Safety (EHS) department to ensure compliance with all WISHA regulations. In addition to this, OSHA literature was also frequently referenced due to its abundance and its applicability to work places across the country.

\section{Prepreg Treater Overview}

The prepreg treater can be broken into 4 major sections: the unwind/bath, ovens, ventilation and take-up, as shown in Figure 1. In the bath section of the machine, fabric is unwound from a roll, dipped into the resin bath and then threaded between two pinch rollers to remove excess resin. From there, the resin-coated fabric is conveyed into the oven section of the machine, where the material is "B-staged". During B-staging, the material is partially cured and solvent is removed, necessitating the use of a ventilation system to capture volatiles. ${ }^{1}$ Finally, the material, now considered prepreg, moves to the take-up assembly where it is cooled and coated with a polyfilm backing, and wound up into a roll for storage.

Movement of the fabric through the oven is controlled by two systems: a closed-loop tensioning system and a speed control system. The closed-loop tensioning system consists of a motor, clutch, load cells and a dead idle roller, and is used to ensure consistent fabric tension throughout the manufacturing process. The speed control system sets and continuously monitors fabric line speed, using a three-phase induction motor, compact power inverter, microcontroller and rotary 
encoder located in the take-up assembly. Using a proportional feedback loop, the speed control maintains a consistent line speed while the take-up roll diameter increases as prepreg is manufactured and rolled up. For more information on the construction of the prepreg treater, see Development of a Solvent-Based Prepreg Treater by Nikki Larson et al. ${ }^{5}$

Though the treater was designed for use with a variety of resin systems, research thus far has focused on the production of prepreg from an alcohol-solvated resole phenolic resin with a fiberglass fabric. Phenolic resin is the reaction product of phenol with formaldehyde to form a three dimensional polymer network, as shown in Figure 2. In the case of a resole phenolic resin, formaldehyde is the excess reactant, and some will remain in the resin system until a high degree of cure

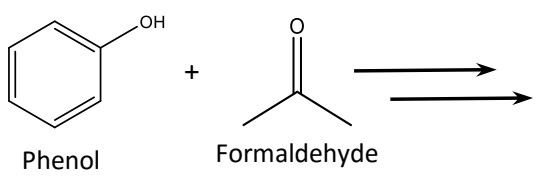

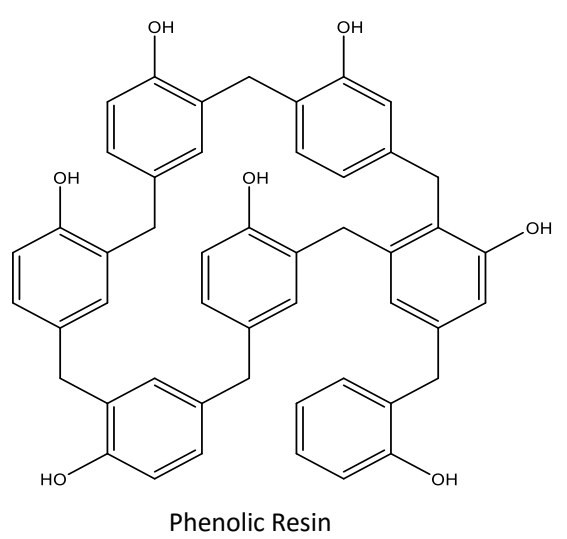

Figure 2. Reaction of phenol and formaldehyde to form phenolic resin has been reached. The solvent used was a mixture of ethanol, methanol and isopropanol. This resin system is processed between 150 to $250^{\circ} \mathrm{F}$, a temperature range under which formaldehyde and alcohol solvents will volatilize, due to their low boiling points. ${ }^{6,7,8}$

\section{Development of Safety Features and Protocol}

\subsection{Safety and Physical Hazards}

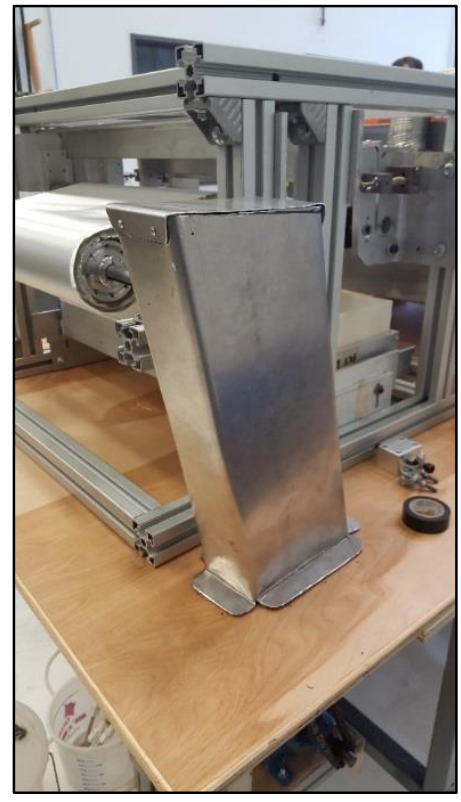

Figure 3. Chain guard constructed to cover tensioning motor
Physical and safety hazards can be defined as "factors within the environment that can harm the body without necessarily touching it" and "unsafe conditions that can cause injury illness and death", respectively. ${ }^{9}$ On the prepreg treater, these are mainly limited to pinch points on the chain drives and heated surfaces of the ovens. To mitigate the risks associated with these dangers, the students constructed aluminum chain guards to cover the moving parts (shown in Figure 3) and selected countertop convection ovens with appropriate safety features.

Additional safety hazards encountered during the construction process included usage of a variety of machinery including mills, lathes, saws, drills, and other power tools. The students received thorough training on each machine before running for the first time, and were supervised throughout usage to minimize risk of injury. This is standard 
practice in the department and is considered adequate protection by WWU's EHS department.

\subsection{Health Hazards}

Health hazards as defined in this paper are a subcategory of OSHA's chemical hazard definition: "[a] chemical for which there is statistically significant evidence based on at least one study conducted in accordance with established scientific principles that acute or chronic health effects may occur in exposed employees." ${ }^{10}$ Using this definition, a number of materials used in this research project can be considered health hazards including the resin itself, volatiles released during the curing process, and the organic solvents used in the manufacturing and cleaning processes. Of these chemicals, formaldehyde can be considered particularly dangerous due to its classification as a sensitizer - "a chemical that causes a substantial proportion of exposed people or animals to develop an allergic reaction in normal tissue after repeated exposure to the chemical". ${ }^{11,12}$ The sensitization of a student to formaldehyde or another chemical has the potential to severely limit their future career, as it would limit the materials with which they could safely work with for the remainder of their life.

Two separate but complimentary approaches were taken to prevent chemical exposures during prepreg manufacturing: the implementation engineering controls in the form of a point of source capture ventilation system and usage of extensive personal protective equipment (PPE). During the construction of the prepreg treater, adequate ventilation levels were determined with the assistance of EHS, by simultaneously measuring volatile organic compound (VOC) concentrations and vacuum levels at the plenum of the ventilation system. Using this method, it was determined that -0.4 in of water provided adequate ventilation to maintain acceptable VOC levels. A negative pressure gauge was installed at the plenum to allow the team to monitor ventilation levels during processing.

Determination of adequate PPE was a lengthier process, as it required manufacturing material and measuring formaldehyde $\left(\mathrm{CH}_{2} \mathrm{O}\right)$ and VOC concentrations in the air at different distances from the machine. A RAE Systems MultiRae Air Monitor was used to measure these values. This instrument was selected for its ability to measure a variety of volatile compounds through the use of different cartridges, which will be useful when the team begins to process additional resin systems.

These measurements allowed the team to select an appropriate respirator and cartridge combination for this resin system. As the highest measured $\mathrm{CH}_{2} \mathrm{O}$ concentration was 5.1 parts per million (ppm), a full-face respirator was required in accordance with OSHA's assigned protection factors (APF). A half-face respirator is certified to provide an $\mathrm{APF}=10$,

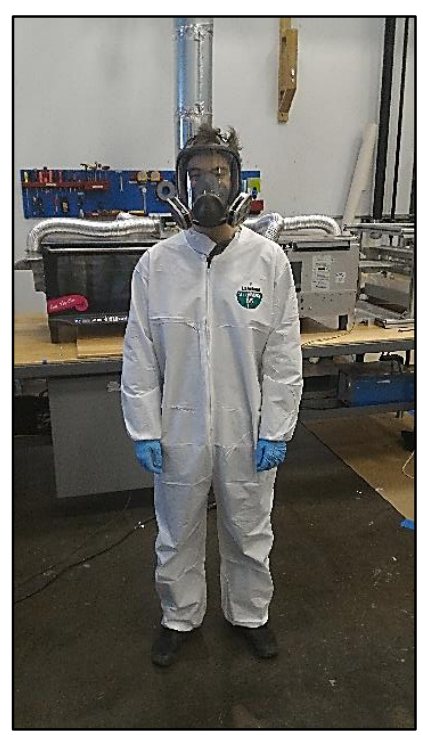

Figure 4. PPE worn by research team while manufacturing prepreg 
indicating it is designed to protect up to 10 times the permissible exposure limit (PEL) of a chemical, which is $0.75 \mathrm{ppm}$ for $\mathrm{CH}_{2} \mathrm{O} \cdot{ }^{13}$ The full-face respirator has an $\mathrm{APF}=50$, which will protect the team to formaldehyde and VOC levels well above those seen during processing. ${ }^{14}$ The respirator cartridge recommended by EHS for use with phenolic resin were 3M 6005 Formaldehyde/Organic Vapor Cartridge. Each set of cartridges is used for 8 hours then replaced, as per manufacturer recommendation. ${ }^{14}$

Table 1. Formaldehyde and VOC measured levels and protection levels

\begin{tabular}{|l|c|c|}
\hline & $\mathrm{CH}_{2} \mathrm{O}(\mathrm{ppm})$ & $\mathrm{VOC}(\mathrm{ppm})^{*}$ \\
\hline Highest Measured Value & 5.1 & 11.3 \\
\hline Full-Face Protection Level $(\mathrm{APF}=\mathbf{5 0})$ & 37.5 & - \\
\hline Half-Face Protection Level $(\mathrm{APF}=\mathbf{1 0})$ & 7.5 & - \\
\hline
\end{tabular}

*No PEL set by OSHA

The team also wears protective layers during processing sessions, including chemical resistant Tychem suits and nitrile gloves, as shown in Figure 4. In the future, it may be necessary to reevaluate these choices, based on the solvents used in processing and clean up procedures. Acetone, a common cleaning solvent, breaks through nitrile gloves almost immediately, thus if the team moves to a resin system necessitating the use of acetone, a new glove material will be needed. ${ }^{16}$ This reevaluation of PPE for new resin systems also applies to respirator and cartridge choices.

\subsection{Fire and Explosion Hazards}

During the initial construction of the prepreg treater, it was assumed that the risk of an explosion or fire was relatively low, given the low concentration of volatiles present in the system at any given time. However, following an incident with a solvent-based treater system at a local aerospace materials manufacturer's facility, new fail-safe features were added to increase machine safety. ${ }^{17}$ These fail-safes were specifically designed to prevent volatile build up in the machine in the event of ventilation or facility power failure - situations in which an unsafe level of volatiles could have previously accumulated. Through consulting with EHS and other faculty and staff within the department, it was determined that the safety features needed to do the following:

- Monitor ventilation to prevent treater from operating without adequate ventilation

- Limit oven temperatures to a safe working range and prevent oven fans from shutting down

- Monitor line movement and prevent material from stagnating in oven

- Remove resin-wet fabric from oven in event of ventilation failure

- Alert users to dangerous situations

- Provide power to oven fans and other safety features in the event of a power failure

The system uses a programmable logic controller (PLC) to monitor and control all safety features. The machine parameters monitored by the PLC are the oven temperatures, ventilation pressure, line speed control, rotary encoder, and oven fan activity. The PLC regulates the startup 
of the machine, ensuring that treater cannot be used until the ventilation system is has achieved a negative pressure of 0.4 in of water, as previously mentioned in Section 3.2. After this minimum value has been reached, the oven fans are turned on by the PLC automatically. At this point, it becomes possible to heat the ovens, as well as control the line speed, allowing the prepreg treater to begin manufacturing material.

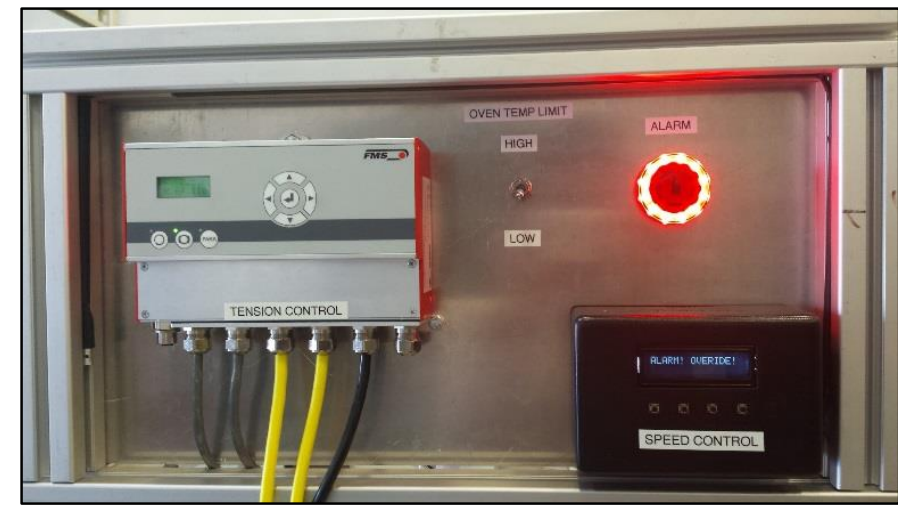

Figure 5. Main control board during alarm state

While the machine is running, the PLC continuously monitors the parameters mentioned above. The following scenarios will trigger an alarm state: inadequate ventilation, loss of power to oven fans, oven temperature above set limit, or unexpected fabric stoppage. If any of these events occur, the alarm will sound and begin flashing red, as shown in Figure 5, to alert users to the danger. When the treater enters an alarm state, the oven heaters will immediately shut off and the line speed will increase to its maximum speed of 9 in per min, to remove material from the oven as quickly as possible, before shutting off automatically after 10 minutes. A backup battery was installed to provide power to the alarm and other safety features in the event of a power failure, to allow for the above emergency shut down procedure to take place. Specifically, the backup battery is intended to power the fans to increase the rate at which volatiles are dispersed to the room, to prevent the volatile build up required for an explosive incident. A flow chart of this process can be seen in Appendix 1.

Work to improve the safety features on the treater is ongoing. While the team believes the current features are a good starting point for preventing explosions, more work is necessary to automate the shutdown procedure when an alarm state occurs. Currently, the roller submerging the fabric into the resin bath must be manually raised before the team can safely exit the facility. Going forward, an automatic release switch will be installed to more fully automate this shut down procedure.

\subsection{Environmental Hazards}

Environmental hazards can be defined as a "state of events which has the potential to threaten the surrounding natural environment and adversely affect people's health". Although the phenolic resins used by the research team thus far are considered relatively ecologically safe, the usage of any material containing organic solvents must be taken seriously, due to their potential to negatively impact the environment. ${ }^{18,19}$

At Western Washington University, EHS handles all chemical waste disposal to streamline the hazardous waste management process and ensure proper storage and disposal procedures followed. Research students are trained to properly collect and label all waste, including 
contaminated solid waste such as resin coated gloves and other PPE. To minimize the risk of spills, secondary containment is always used when storing and transporting materials, and the amount of chemicals stored on site is kept to a minimum.

\subsection{On-Going Safety Training}

Safety training is an essential component of any safety system - without proper training, effective usage of the safety features and protocol cannot be ensured. All students in the Engineering and Design Department take an annual general laboratory safety training class to review proper laboratory procedures [and other things] as well as online chemical safety training, which instructs them on proper chemical handling, disposal and understanding safety data sheets (SDS). Students receive further safety training on specific machinery as required... All students using respirators receive yearly fit checks. For those using full-face respirators, an additional check is required to ensure proper fit.

Prior to operating WWU's treater, the students receive training on operation of the prepreg treater and its safety systems from an involved faculty or staff member. This training includes troubleshooting during an alarm state, recognizing dangerous situations, and evacuation procedures. Hard copies of the procedures for operation of the treater safety systems are also available for reference at the facility in which the prepreg treater is located. Additionally, a faculty or staff member is always present during prepreg manufacturing, as an additional security measure.

\subsection{System Assessment}

WWU's EHS has been essential for assessing the safety of the prepreg treater at all stages of the research project, from the design and construction of the machine onwards. With their assistance, the WWU team has been able to ensure compliance with university and WISHA regulations. When new chemicals/resins are introduced they are reviewed by the chemical safety officer. Once they are deemed acceptable the team then confers with EHS to determine the most appropriate personal protective equipment to work safely with the new system

The team also internally assesses safety when changes are made to either the machine or the processing procedure and/or materials. It is a goal of the involved faculty to instruct students on proactive vs reactive thinking when making research decisions, and to incorporate this into group decision-making whenever possible. Due to the undergraduate nature of the research, the level of turnover in group members is relatively high. While this necessitates more frequent trainings and slows the pace at which progress is made, it also provides frequent opportunities to gain fresh perspectives on the system and policies in place. Students are highly encouraged to provide not only feedback and suggest improvements to the system, but to remain vigilant around the subject of safety in general.

\section{Summary}


As safety is of the utmost concern while working in any academic or manufacturing workplace environment, the research team has prioritized the creation and maintenance of a safe work environment above all else. Since its inception, the team has made great improvements to the safety features and protocol to address safety concerns. As updates to the machine continue to be made and new resin systems are introduced, the safety features and protocol will be continually reevaluated to ensure the safety of all users.

\section{References}

1. Heth, J. High-Performance Composites. June 2000, pp. 32-36.

2. A Guide to Workplace Safety and Health in Washington State; F416-132-000; Washington State Department of Labor \& Industries, 2013.

3. About WISHA, DOSH, and OSHA. A-Z Safety \& Health Topics, http://www.lni.wa.gov/safety/topics/atoz/about/default.asp (accessed Jan 31, 2016).

4. Frequently Asked Questions. Directorate of Cooperative and State Programs | State Plans, https://www.osha.gov/dcsp/osp/index.html (accessed Jan 31, 2016).

5. Larson, N.; Rasnack, W.; Hoekstra, N.; Boland, C.; Leone, E.; Santos, I.; Healy, K. R.; Chawla, T. S.; Schoepe, S. Development Of a Solvent-Based Prepreg Treater. In 2015 ASEE Annual Conference And Exposition; American Society of Engineering Education: Seattle, 2015.

6. Gardziella, A.; Pilato, L.; Knop, A. Phenolic Resins: Chemistry, Applications, Standardization, Safety, and Ecology; 2nd ed.; Springer: Berlin, 2000.

7. Haynes, W.M. CRC Handbook of Chemistry and Physics. 91st ed. Boca Raton, FL: CRC Press Inc., 20102011.

8. O'Neil, M.J. The Merck Index - An Encyclopedia of Chemicals, Drugs, and Biologicals. Whitehouse Station, NJ: Merck and Co., Inc., 2006.

9. OSHA Hazards, https://www.osha.gov/dte/grant_materials/fy10/sh-20839-10/circle_chart.pdf (accessed Jan 31, 2016).

10. Hazardous Chemical Definition, http://www.research.northwestern.edu/ors/safety/hazardcommunication/definition.html (accessed Jan 31, 2016).

11. NIOSH Skin Notation Profiles Formaldehyde/Formalin; Department of Health and Human Services: Cincinnati, OH, 2011.

12. Occupational Safety \& Health Administration. Code of Federal Regulations, Part 1917.28 App A, Title 29, 1983.

13. Occupational Safety \& Health Administration. Code of Federal Regulations, Part 1910.1048, Title 29, 1992.

14. Assigned Protection Factors for the Revised Respiratory Protection Standard; OSHA 3352-02;

Occupational Safety and Health Administration, Washington, DC 2009.

15. Cartridge and Filter Guide; 3M Personal Safety Division: St.Paul, MN, 2014.

16. Seventh Edition Ansell Chemical Resistance Guide Permeation \& Degradation Data; Technical Report for Ansell Occupational Healthcare: Coshocton, OH, 2003.

17. Smith, A.; Walters, S. Aerospace Plant Explosion Rattles Homes Two Miles Away. NBC News, http://www.nbcnews.com/news/us-news/explosion-washingtons-zodiac-aerospace-plant-causes-injuriesn392341 (accessed Jan 31, 2016).

18. Toxicological Profile for Phenol; U.S. Department of Health and Human Services: Atlanta, GA, 2006. 
19. Bredenberg, A. Reducing the Environmental Impact of Industrial Solvent Use. Industry News, http://news.thomasnet.com/imt/2012/06/11/reducing-the-environmental-impact-of-industrial-solvent-use (accessed Jan 31, 2016). 


\section{Appendix 1}

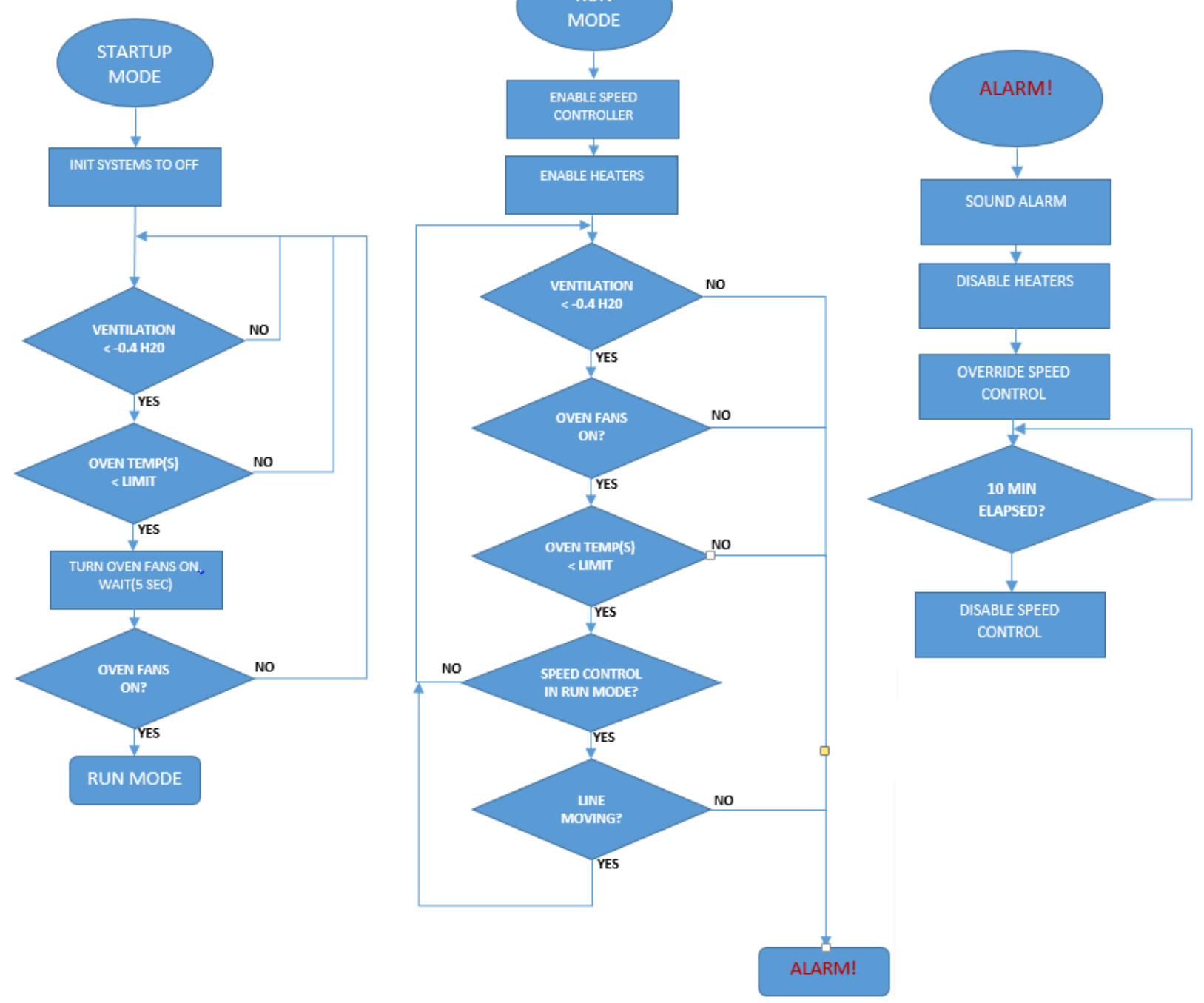

Figure 6. Flow chart of safety feature control system during startup mode, run mode, and in an alarm state 